\title{
In-silico Investigation of the Interaction between Beta-class Glutathione S-Transferase and Five Antibiotics, namely; Ampicillin, Tetracycline, Chloramphenicol, Ciprofloxacin and Cephalexin
}

\author{
${ }^{*}$ SHEHU, D; ${ }^{2}$ DANLAMI, S; ${ }^{1}$ YA'U, M; ${ }^{1}$ BABANDI, A; ${ }^{1}$ YAKASAI, HM; \\ 1BABAGANA, K
}

\author{
${ }^{*}$ Department of Biochemistry, College of Health Sciences, Bayero University, Kano, PMB 3011, Kano Nigeria \\ ${ }^{2}$ Department of Nursing Science, Kaduna state University, Kaduna \\ *Corresponding Author Email: dshehu.bch@buk.edu.ng
}

\begin{abstract}
Glutathione s-transferases(GSTs) are enzymes involved in the conjugation and deactivation of various xenobiotics including drugs. Thisin-silico study was undertaken in order to investigate the interaction between beta-class glutathione s-transferase and five selected antibiotics, namely; ampicillin, tetracycline, chloramphenicol, ciprofloxacin and cephalexin using molecular docking study. RaptorX server was used to predict the amino acids involved at the binding sitewhile molecular docking study was employed in order to investigate the binding interactions.RaptorX predicted several amino acids which were different from the ones observed in molecular docking because of the variability in the substrate binding site of GSTs however, all the amino acids predicted by RaptorX were also found to be involved in the GSH binding.Lys107, Phe109, Ser110, Leu113, Trp114, His115 and Arg123, Leu168 were the amino acids involved in the binding of various antibiotics to the substrate binding site of the protein while Ala9, Cys10, Leu32, Tyr51, Val52, Pro53, Glu65 and Ala66were involved in the binding of the co-substrate GSH to the binding site of the protein. The results indicated that all the antibiotics showed a good binding affinity with the beta class GST and are therefore capable of deactivating the drugs. With these, finding a beta class GST inhibitors alongside antibiotics during a treatment of diseases will be of beneficial in the current fight against antibiotic resistance.
\end{abstract}

DOI: https://dx.doi.org/10.4314/jasem.v25i4.2

Copyright: Copyright $(\odot 2021$ Shehu et al. This is an open access article distributed under the Creative Commons Attribution License (CCL), which permits unrestricted use, distribution, and reproduction in any medium, provided the original work is properly cited.

Dates: Received: 12 December 2020; Revised: 26 January 2021; Accepted: 12 February 2021

Keywords:Beta class, Glutathione s-transferase, Antibiotics, Molecular docking, Resistance

Antibiotic resistance is a global phenomenon threatening the efficacy of several antibiotics that saved the lives of millions people worldwide(Frieri et al., 2017). While this phenomenon is attributed to the indiscriminate use of antibiotics, however, drugs metabolizing enzymes are playing a great role due to increase in their expression level and subsequent deactivation of the drugs (Hattinger and Serra, 2015). Other factors such as inappropriate prescription, extensive agricultural use, availability of few antibiotics and regulatory barriers are also playing a great role in the development of antibiotic resistance (Unjiya, 2017). Glutathione s-transferase (GSTs) is a detoxification enzyme found in phase II of xenobiotics metabolism(Alias and Clark, 2010). The enzyme plays a great role in the conjugation of several drugs and other xenobiotics rendering them much more water soluble that can be easily excreted(Shehu, et al., 2019). While the enzyme is beneficial in the detoxification of several toxins that can be harmful to living organisms at the same time, the detoxification of several drugs which in other ways serve to save the lives of the organism is threatening the existence of human race as a whole (Arca et al., 1988; Morrow et al., 1998). This prompted a lot of researches on inhibitors of GST especially during treatment of certain diseases such as cancer(Allocati et al., 2018).Several different isoenzymes of GST have been identified in bacteria including Beta, Chi, Zeta, Rho, $\mathrm{Nu}$, and Theta classes(Shehu et al., 2019). Genome sequencing of several pathogenic bacteria reveals the presence of different classes of these GSTs (Shehu et al., 2019). Among these classes of isoenzymes, beta class is specific to bacteria and is the one so far investigated to have a conjugation function against ampicillin (Allocati et al., 2000). However, the nature of the interaction of this GST class with the antibiotic is unknown. Furthermore, the possibility of conjugating other antibiotics by this class of GST is not known. Molecular docking studies is one of the rapidly and in-silico model for investigating the interaction between enzymes and various ligands. We recently cloned and characterized a beta class GST, termed KKS-BphK from a polychlorobiphenyl degrading organism; Acidovorax sp. KKS102 (Shehu and Alias, 2018). In this study, in-silico analysis was 
used to investigate the interaction between beta-class glutathione -transferase and five selected antibiotics, namely; ampicillin, tetracycline, chloramphenicol, ciprofloxacin and cephalexin using molecular docking study.

\section{MATERIALS AND METHODS}

Materials: Amino acid sequence of BphK-KKS from (Acidovorax sp. KKS102): The amino acid sequence of BphK-KKS was retrieved from the NCBI database and used to model the protein for molecular docking studies using swiss model workspace (https://swissmodel.expasy.org/interactive).

Substrates (Antibiotics used): The substrate used were; Ampicillin,Tetracycline, Chloramphenicol, Cephalexin and Ciprofloxacin.

Methods:Sequence alignment analysis: Amino acid sequence of KKS-BphK and glutathione s-transferase from other organisms were downloaded from NCBI database and were sent in fasta format to the clustalW server for sequence alignment analysis (Thompson et al., 2003). The figure for the sequence alignment study was produced using Jalview software(http://www.ebi.ac.uk/ michele/jalview/cont ents.html).

Binding site Prediction:The FASTA format of KKSBphK sequence was submitted into the RaptorX binding site prediction server (http://raptorX.uchicago.edu/Binding) which automatically modeled the protein and predict its binding site(Källberg et al., 2012).

Molecular Docking:A free accessible software autodock 4.2 was used for the molecular docking studies (Goodsell et al., 1996). Using the amino acid sequence retrieved from NCBI database, the threedimensional structure of KKS-BphK was built using swiss model server (Bordoli et al., 2009). The structures of all the ligands under study (mol2 file) were constructed and optimized using Chemsketch software. The mol 2 files were then used as input for Open babel software to generate the protein data bank (PDB) file of all the ligands (O'Boyle et al., 2011). The PDF files of both the protein and ligands were used as input files in autodock tools (ADT). Polar hydrogen, Kollman charges and solvation parameters were added to the protein while rotatable bonds for the ligands were defined. The prepared files were saved in the form of pdbqt format. Because of the variability in the substrate binding site of GSTs, a blind docking analysis was set up using an autogrid size of 126,126 and 126 for the $\mathrm{x}, \mathrm{y}$ and $\mathrm{z}$-axis respectively. Cygwin software was used in running the docking algorithm. A total of 100 runs were made for each binding site using Lamarckian genetic algorithm as search engine. The parameters used in auto-docking process were as follows: GA population size $=100$; maximum number of energy evaluations $=250,000$. Default parameters were used for mutation, crossover and elitism. Cluster analysis was performed on the docked result using a root mean square deviation (rmsd) of $2 \AA$. The docked conformations were visualized using Discovery studio software.

\section{RESULTS AND DISCUSSION}

GSTs contained two binding sites with different amino acids participating in the interaction of ligands to these binding sites. The amino acids responsible for the binding of the ligands are located at the C-terminal part of the protein while $\mathrm{N}$-terminal site contained amino acids responsible for the binding of the cosubstrate; GSH (Shehu and Alias, 2018).The result of binding site prediction using RaptorX indicated the preferred ligand of KKS-BphK to be GSH (Table 1). Other ligands cannot be predicted simply because the C-terminal part of the protein was designed to accommodate different types of substrates (Shehu et al., 2019). Pocket multiplicity, which represent the frequency with which the selected pocket was found in the template structures were found to be 91 and 69 (Table 1). This indicated a very good prediction of the ligand binding sites as any pocket multiplicity above 40 represent a good chance of true predicted pocket. Beta class GST is characterized by cysteine residue at position 10 in the $\mathrm{N}$-terminal region of the protein (Allocati et al., 2000). The result of sequence alignment study of representatives of beta class GST from various organisms also showed that they all contained cysteine residue at that position (Fig 1), which also correspond to the prediction made by RaptorX. More so, the sequence alignment also indicated the presence of several conserved amino acids at the N-terminal region responsible for $\mathrm{GSH}$ binding. Furthermore, the $\mathrm{C}$-terminal region was also found to contain certain conserved amino acids which were shown to be critical in the binding of various ligands (Allocati et al., 2012). Comparative analysis between the predicted binding pocket amino acids and sequence alignment study showed that C10, V52, P53, H106, K107 and W164 were all perfectly conserved in all the beta class GSTs analyzed (Fig 1). Other predicted amino acids were relatively less or not conserved at all between the beta class GSTs.The result of the molecular docking indicated that several amino acids both in the substrate binding site and glutathione binding site played a great role in the binding and subsequent transformation of the substrates.Lys107, Phe109, Ser110, Leu113, Trp114, His115, Arg123 and Leu168, are the major amino acids involved in the binding of various ligands at the 
substrate binding site (Fig. 2-7). Among these amino acids, only Lys107 was found to be predicted using RaptorX. This may explain the reason why the Nterminal domain of GSTs accommodate different types of substrates because of their variability in the amino acids at the substrate binding site. Several other amino acids also played a role in the binding of the cosubstrate GSH to the binding pocket of KKS-BphK. This includes; Ala9, Cys10, Leu32, Tyr51, Val52, Pro53, Glu65 and Ala66 (Fig. 2-7). In contrast to the amino acids at the $\mathrm{C}$-terminal part of the protein, all the amino acids at the N-terminal part which is the GSH binding site were predicted by RaptorX server. The binding is mediated using different types of bondings including; hydrogen, ionic and hydrophobic interactions.

Table 1.Predicted Binding pocket and Binding site residues of

\begin{tabular}{llll} 
& \multicolumn{3}{c}{ KKS-BphKby RaptorX } \\
\hline & $\begin{array}{l}\text { Pocket } \\
\text { multiplicity }\end{array}$ & $\begin{array}{l}\text { Top } \\
\text { ligands }\end{array}$ & Binding residues \\
\hline 1 & 91 & GSH & A9, C10, L32, Y51, V52, P53, \\
& & & E65, A66, H106, K107, W164 \\
2 & 69 & GSH & T99, T103, E104 \\
\hline
\end{tabular}

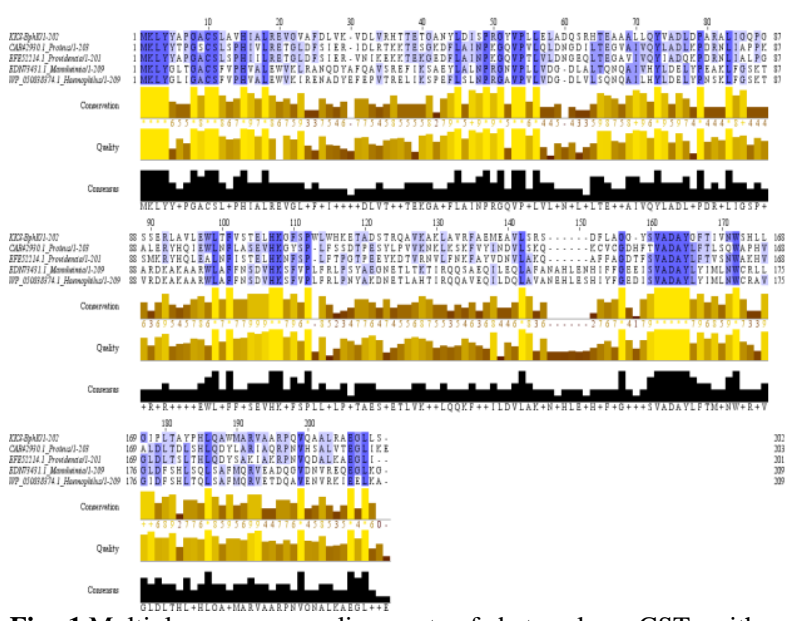

Fig 1.Multiple sequence alignment of beta class GST with representatives from various organisms. KKS-BphK is the reference GST from Acidovoraxsp KKS 102, CAR42930.1 is from Proteus mirabilis HI4320, EFE52214.1 is from Providenciarettgeri DSM 1131, and EDN73431.1 is from Mannheimiahaemolytica PHL213, WP_050838374.1 is from Haemophilus influenza. Alignment was constructed using ClustalW in Jalview.

\begin{tabular}{lllll} 
& \multicolumn{2}{l}{ Table 2.Summary of Binding interactions of different antibiotics with KKS-BphK } \\
\hline S/NO & Substrates & $\begin{array}{l}\text { Minimum } \\
\text { Binding Energy }\end{array}$ & $\begin{array}{l}\text { Number of } \\
\text { Hydrogen Bonds }\end{array}$ & $\begin{array}{l}\text { Amino acids involved in } \\
\text { the binding }\end{array}$ \\
\hline 1 & Ampicillin & -9.26 & 4 & His115, Arg123, \\
2 & Tetracycline & -10.65 & 3 & Trp114, His115 \\
3 & Chloramphenicol & -9.18 & 2 & Lys107 \\
4 & Ciprofloxacin & -8.92 & 2 & Lys107 \\
5 & Cephalexin & -9.61 & 8 & His115, Arg123, Phe109, \\
& & & & Trp114 \\
\hline
\end{tabular}

In the molecular docking study, cluster analysis was performed for all the docked results using root mean square deviation (RMSD) of $2 \AA \hat{\text {. }}$. Moreover, the number of hydrogen bonds and the amino acids involved were all presented (Table 1). The top-ranked pose with lowest docked binding affinities are generally used as a standard selection in most docking programs. The result using ampicillin as a substrate reveals 13 different conformations. The lowest minimum binding energy obtained was -9.26 $\mathrm{Kcal} / \mathrm{mol}$, this occurred in the $56^{\text {th }}$ run of the cluster containing 33 members. Four (4) hydrogen bonds were found to be involved with His 115 and Arg 123 contributing to the formation of the bond (Fig 2). The result of molecular docking study using tetracycline as substrate reveals 11 different conformations with the lowest minimum binding energy of $-11.69 \mathrm{Kcal} / \mathrm{mol}$ obtained in the $60^{\text {th }}$ run of a cluster containing 16 members. Three (3) hydrogen bonds were involved using Trp 114 and His 115 (Fig 3). Using chloramphenicol as a substrate, the result showed a minimum binding energy of $-9.18 \mathrm{Kcal} / \mathrm{mol}$ which was obtained in the $66^{\text {th }}$ run of the cluster containing 8 members. Lys 107 was the only amino acid here involved in the formation of two (2) hydrogen bonds
(Fig 4). Both ciprofloxacin and cephalexin were found to have minimum binding energies of $-8.92 \mathrm{Kcal} / \mathrm{mol}$ and $-9.61 \mathrm{Kcal} / \mathrm{mol}$ respectively. These were obtained in the $56^{\text {th }}$ run of a cluster containing 11 members and $10^{\text {th }}$ run of a cluster containing 33 members respectively.

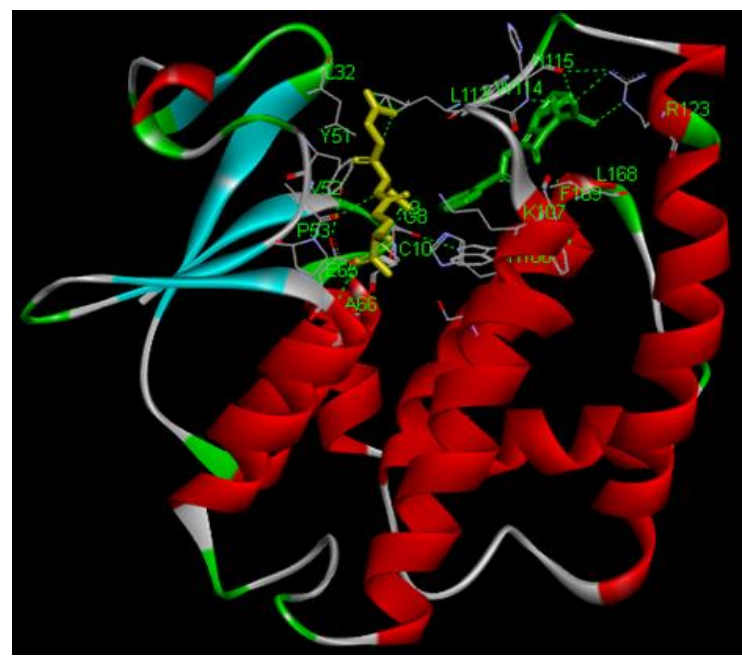

Fig 2. Binding interaction of KKS-BphK with ampicillin, the yellow ball and stick presentation represent the co-substrate GSH while the green is the ampicillin substrate 


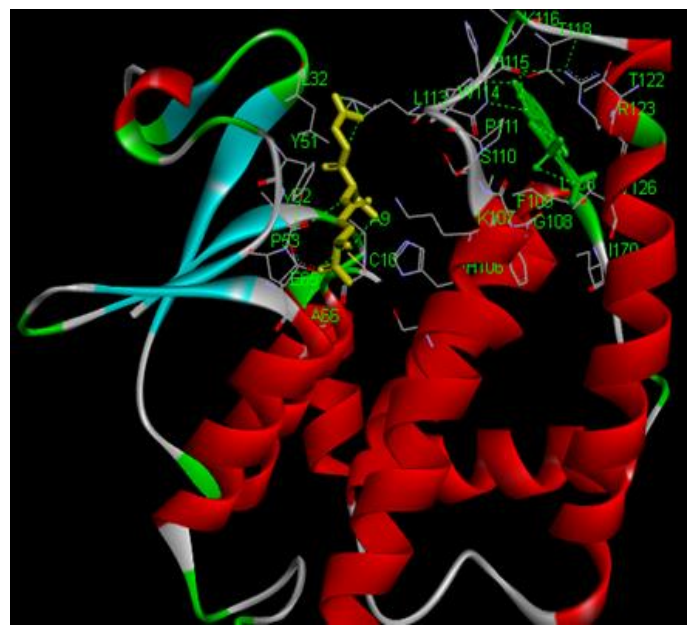

Fig 3. Binding interaction of KKS-BphK with Tetracycline, the yellow ball and stick presentation represent the co-substrate GSH while the green is the ampicillin substrate.

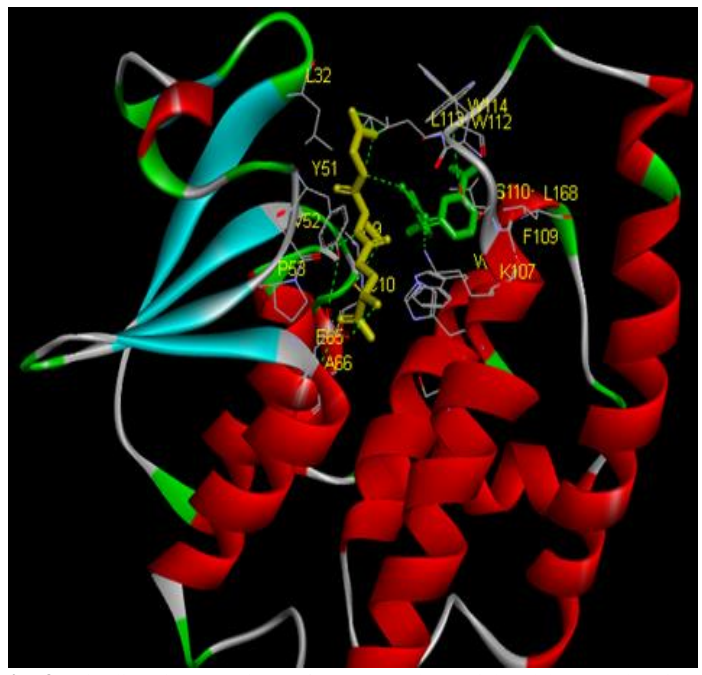

Fig 4. Binding interaction of KKS-BphK with Chloramphenicol, the yellow ball and stick presentation represent the co-substrate GSH while the green is the ampicillin substrate

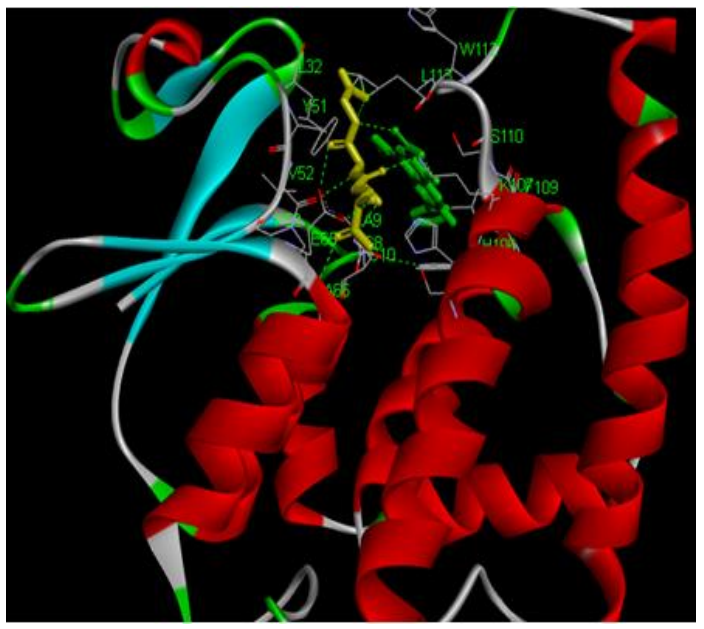

Fig 5. Binding interaction of KKS-BphK with Ciprofloxacin, the yellow ball and stick presentation represent the co-substrate GSH while the green is the ampicillin substrate

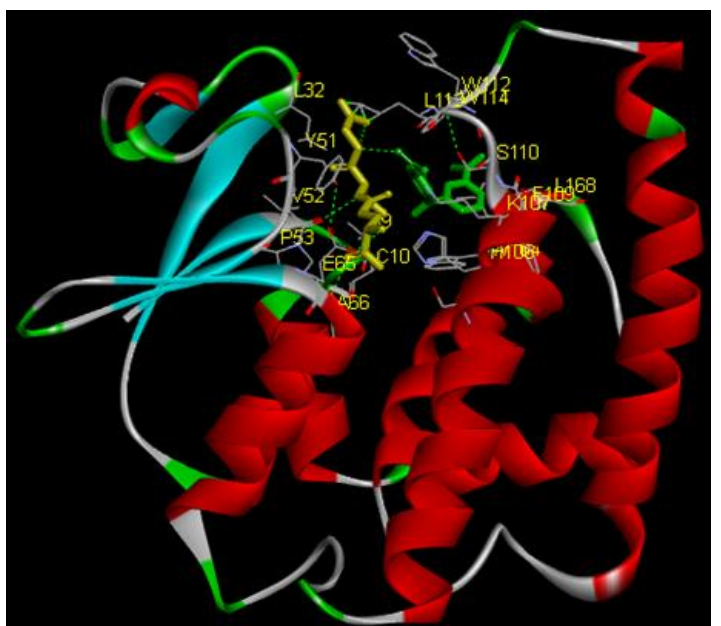

Fig 6. Binding interaction of KKS-BphK with Cephalexin, the yellow ball and stick presentation represent the co-substrate GSH while the green is the ampicillin substrate.

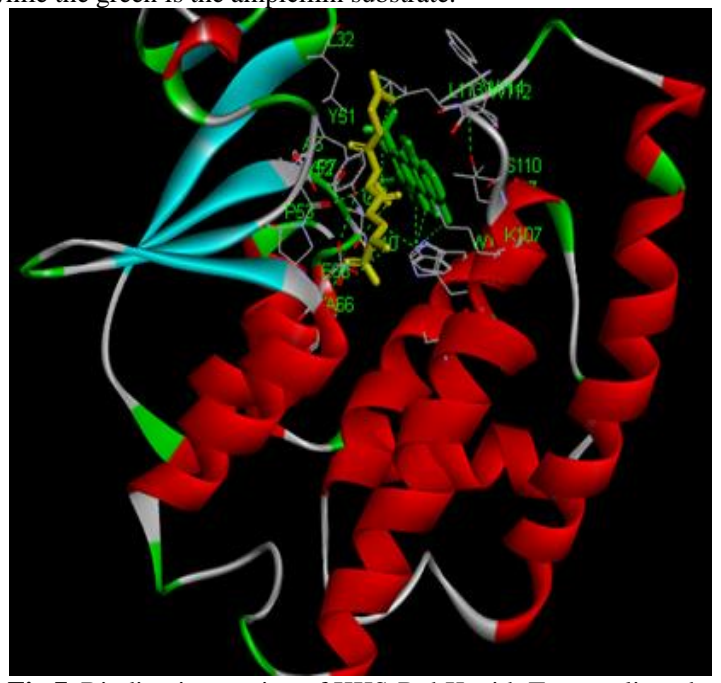

Fig 7. Binding interaction of KKS-BphK with Tetracycline, the yellow ball and stick presentation represent the co-substrate GSH while the green is the ampicillin substrate.

Two (2) and Eight (8) hydrogen bonds were found to mediate the interaction of ciprofloxacin and cephalexin respectively. The two hydrogen bonds were formed via Lys 107 while His115, Arg123, Phe109, Trp114 contributed to the formation of eight hydrogen bonds (Fig 5 and Fig 6) respectively. However, even though -11.69 is the lowest minimum binding energy obtained from interaction of tetracycline with KKS-BphK, but the interaction of the ligand does not seems to occur in the KKS-BphK binding pocket. The second lowest minimum binding obtained was -10.65 which form the best correct docking pose with the protein (Fig 7).The molecular docking studywas carried out in order to explore the possibility of binding interactions between KKSBphK with various antibiotics. Molecular docking study nowadays emerges as a novel tool of studying interactions of various proteins with ligands which 
helps to unravel the structure-function relationship and in rational drug design(Mavromoustakos et al., 2011). Glutathione s transferases are phase II detoxification enzymes which are known to play a vital role in the detoxification of various xenobiotics in the body system(Alias and Clark, 2010). The function served a crucial purpose of keeping biological systems safe from the toxic effect of many xenobiotics, however, in other organisms such as bacteria and many other pathogenic organisms, the system helps in accelerating resistance to various chemotherapeutic agents thereby helping the pathogens to thrive in the body system(Ang et al., 2005; Li et al., 2017). Previous studies have shown that Beta class glutathione stransferase played a great role in the defense of bacterial cells against some antibiotics and other stress causing compounds(Allocati et al., 2000; Arca et al., 1988; Pugazhendhi et al., 2017). Based on this study, tetracycline with minimum binding energy of -11.62 may likely induce expression of beta class GST and therefore be deactivated by beta class GST more than the other compounds under study. This is based on the fact that; the lower the minimum binding the higher the tendency of binding of a particular compound to its target protein (Mavromoustakos et al., 2011). Therefore, based on this study, the order of binding of these antibiotics to $\mathrm{KKS}-\mathrm{BpHk}$ is tetracycline> cephalexin> ampicillin> chloramphenicol > ciprofloxacin.

Conclusion:In conclusion, five different antibiotics were employed in order to investigate the possibility of binding of these antibiotics to GSTs.All the antibiotics showed a good binding affinity to the binding site of the protein and therefore suggest the possibility of binding and deactivation of these drugs by the Beta class GST. Therefore,the study can serve as a clue forfuture research and inclusion of beta class GST inhibitors in the current fight against antibiotic resistance.

\section{REFERENCE}

Alias, Z; Clark, AG (2010). Adult Drosophila melanogaster glutathione S-transferases: Effects of acute treatment with methyl parathion. Pestic. Biochem. Physiol. 98(1): 94-98

Allocati, N; Casalone, E; Masulli, M; Polekhina, G; Rossjohn, J; Parker, MW; Carmine, D; (2000). Evaluation of the role of two conserved active-site residues in Beta class glutathione S-transferases. Biochem. J. 351(2): 341-346.

Allocati, N; Federici, L; Masulli, M; Di Ilio, C (2012). Distribution of glutathione transferases in
Gram-positive bacteria and Archaea. Biochimie. 94(3):588-596.

Allocati, N; Masulli, M; Di Ilio, C; Federici, L (2018). Glutathione transferases: substrates, inihibitors and pro-drugs in cancer and neurodegenerative diseases. Oncogenesis. 7(1): 1-15.

Ang, WH; Khalaila, I; Allardyce, CS; JuilleratJeanneret, L; Dyson, PJ (2005). Rational design of platinum (IV) compounds to overcome glutathione-S-transferase mediated drug resistance. J.Am. Chem. Soc. 127(5): 1382-1383.

Arca, P; Rico, M; Brana, A; Villar, CJ; Hardisson, C; Suarez, J (1988). Formation of an adduct between fosfomycin and glutathione: a new mechanism of antibiotic resistance in bacteria. Antimicrob. Agents chemother. 32(10): 1552-1556.

Bordoli, L; Kiefer, F; Arnold, K; Benkert, P; Battey, J; Schwede, T (2009). Protein structure homology modeling using SWISS-MODEL workspace. Nature protocols. 4(1): 1-13.

Frieri, M; Kumar, K; Boutin, A (2017). Antibiotic resistance. J Infection Public Heal. 10(4): 369-378.

Goodsell, DS; Morris, GM; Olson, AJ (1996). Automated docking of flexible ligands: applications of AutoDock. J. Mol. Recognit. 9(1): 1-5.

Hattinger, CM; Serra, M (2015). Role of pharmacogenetics of drug-metabolizing enzymes in treating osteosarcoma. Expert Opin Drug Metab Toxicol. 11(9): 1449-1463.

Källberg, M; Wang, H; Wang, S; Peng, J; Wang, Z; $\mathrm{Lu}, \mathrm{H} ; \mathrm{Xu}, \mathrm{J}$ (2012). Template-based protein structure modeling using the RaptorX web server. Nat. Protoc. 7(8) :1511-1522.

Li, S; Li, C; Jin, S; Liu, J; Xue, X; Eltahan, AS; Liang, XJ (2017). Overcoming resistance to cisplatin by inhibition of glutathione S-transferases (GSTs) with ethacraplatin micelles in vitro and in vivo. Biomaterials. 144:119-129.

Mavromoustakos, T; Durdagi, S; Koukoulitsa, C; Simcic, M; Papadopoulos, M; Hodoscek, M.; Golic Grdadolnik, S (2011). Strategies in the rational drug design. Curr. Med. Chem. 18(17): 2517-2530.

Morrow, CS; Smitherman, PK; Diah, SK; Schneider, E; Townsend, AJ (1998). Coordinated Action of 
Glutathione S-Transferases (GSTs) and Multidrug Resistance Protein 1 (MRP1) in Antineoplastic Drug Detoxification Mechanism of GST a1-1-and mrp1-Associated Resistance to Chlorambucil in MCF7 Breast Carcinoma cells. J. Biol. Chem. 273(32): 20114-20120.

O'Boyle, NM; Banck, M; James, CA; Morley, C; Vandermeersch, T; Hutchison, GR (2011). Open Babel: An open chemical toolbox. J. Cheminformatics. 3(1): 1-4.

Pugazhendhi, A; Dhanarani, S; Shankar, C; Prakash, P; Ranganathan, K; Saratale, RG; Thamaraiselvi, K (2017). Electrophoretic pattern of glutathione Stransferase (GST) in antibiotic resistance Grampositive bacteria from poultry litter. Microb Pathog. 110: 285-290.
Shehu, D; Abdullahi, N; Alias, Z (2019). Cytosolic Glutathione S-transferase in Bacteria: A Review. Pol. J. Environ. Stud. 28(2): 1-6.

Shehu, D; Alias, Z (2018). Dechlorination of polychlorobiphenyl (PCB) degradation metabolites by a recombinant glutathione transferase from Acidovorax sp. KKS102. FEBS Open Bio. 9(3) : 1-8.

Shehu, D; Alias, Z (2018). Functional Role of Tyr12 in the Catalytic Activity of Novel Zeta-like Glutathione S-transferase from Acidovorax sp. KKS102. Protein j. 37(3): 261-269.

Thompson, JD; Gibson, TJ; Higgins, DG (2003). Multiple sequence alignment using ClustalW and ClustalX. Curr protoc Bioinformatics. 1 (2): .3-22.

Unjiya, A (2017). Antibiotic Resistance: A Global Threat. J Adv Med Dent Scie Res. 5(11): 68-71. 